\title{
Prácticum virtual en Educación Física: entre pandemia e incertidumbre Virtual prácticum in Physical Education: between the pandemic and uncertainty
}

Franklin Castillo-Retamal, Lissa Silva-Reyes, Miguel Muñoz-González, Lorena López-Toro, Paulina PlazaCofré, Francisca Arredondo-Muñoz, César Faúndez-Casanova

Universidad Católica del Maule (Chile)

\begin{abstract}
Resumen. La situación sanitaria actual ha obstaculizado muchos procesos formativos de modalidad presencial impactando en las Prácticas de Síntesis Profesional (PSP), provocando que estudiantes finalicen su Proceso Formativo (PF) en la virtualidad. La investigación tuvo como objetivo conocer la percepción de estudiantes de Educación Física (EF) frente a la PrácticaVirtual de Síntesis Profesional. La metodología tuvo un enfoque cuantitativo y la recolección de los datos fue a través de una encuesta de tipo descriptiva, aplicada a 47 egresados de EF de una universidad chilena durante el 2020. Se concluye que los egresados se encontraban preparados para los cambios que se presentaron durante la crisis sanitaria, aceptando la situación dada este año y adaptándose a ella, la cual debe considerarse como una oportunidad de innovación de la asignatura.
\end{abstract}

Palabras clave: Práctica de Síntesis Profesional;TIC; Proceso Formativo; Enseñanza-aprendizaje.

\begin{abstract}
The current health situation has hampered many face-to-face training processes, affecting Professional Synthesis Practices (PSP) and causing students to complete theirTraining Process (TP) in virtually.The objective of the investigation was to know the perception of students regarding theVirtual Practice of Professional Synthesis. The methodology had a quantitative approach and the data was collected through a descriptive survey, applied to 47 Physical Education (PE) teachers who graduated from a Chilean university during 2020. It is concluded that the graduates were prepared to the changes that occurred during the health crisis, accepting the situation given this year and adapting to it, which should be considered as an opportunity for innovation in the subject.
\end{abstract}

Keywords: Professional Synthesis Practice; ICT; Formative Process; Learning-Teaching.

\section{Introducción}

Las PSP en los planes de estudio a nivel universitario constituyen un importante referente de formación, las cuales buscan desarrollar diversas competencias relacionadas con el proceso de enseñanza/aprendizaje, que están vinculadas a los tres saberes de la educación: el saber, el saber ser y el saber hacer (Martínez \& González, 2009). De igual manera, el Marco para la Buena Enseñanza propuesto por el Ministerio de Educación de Chile (MINEDUC, 2008), contiene cuatro dominios enmarcados, a saber, el dominio A (preparación de la enseñanza), dominio B (creación de un ambiente propicio para el aprendizaje), dominio C (enseñanza para el aprendizaje de todos los estudiantes) y dominio D (responsabilidades profesionales) que permiten enlazar lo teórico con la práctica. En esta lógica, la práctica (prácticum) es el eje articulador de todas las actividades curriculares del PF y tiene como objetivo acercar al profesor en formación a la realidad profesional como así

Fecha recepción: 16-01-21. Fecha de aceptación: 28-05-21

Franklin Castillo-Retamal

fcastillo@ucm.cl mismo ayudar en la construcción y concepción del rol docente (Ávalos, 2002; Bisconsini y Oliveira, 2018; Souza de Carvalho et al., 2020).

De acuerdo con esto, las PSP de EF desarrolladas en la universidad estudiada, se mantienen como un eje primordial y específico dentro de los conocimientos que los estudiantes de la carrera deben aprender, existiendo un proceso como tal en todos los semestres impartidos, los que buscan aplicar sus conocimientos en contextos prácticos de acción desde el inicio hasta el final de su formación profesional, permitiendo integrar los distintos saberes, capacidades y desempeños profesionales que necesitará a lo largo de todo su desarrollo como docente (Castillo et al., 2019). Es por esto que el desarrollo de una buena práctica está íntimamente ligado al PF, pues coloca en ejercicio todo lo aprendido durante el transcurso del itinerario formativo, donde el estudiante tiene la oportunidad de vivenciar con anterioridad lo que enfrentará en su futuro profesional (Silva, et al., 2018).

Las prácticas son un hito importante y esencial dentro de todos los procesos formativos de la educación superior, ya que buscan acercar a los estudiantes a las diversas realidades profesionales a las que se verán en- 
frentados una vez finalizados sus estudios y, a su vez, evaluar si las competencias han sido adquiridas a lo largo del proceso, vale decir, el interés de las prácticas pedagógicas radica en que a su vez son una práctica social que se da en un momento concreto y en condiciones particulares (Chamorro, et al., 2008; Anversa et al., 2020; Castillo, et al., 2020).

La situación sanitaria a nivel mundial durante el año 2020 ha obstaculizado todos los procesos de práctica de modalidad presencial en la mayoría de las carreras impartidas en el país, por lo cual, las metodologías han tenido que ser modificadas. Esto ha impactado directamente en las PSP de los estudiantes de pedagogía en EF, teniendo que finalizar su PF de manera diferente a como era normalmente realizada, o bien, suspenderlo hasta retornar a los establecimientos (Flores-Ferro et al., 2021; Almonacid et al., 2021a).

Debido a la facilidad de contagio y a la gravedad con la que ha sido clasificada, se ha optado por el confinamiento de la mayoría de la población y se han suspendido varias actividades presenciales que eran parte de la normalidad, siendo el sistema educativo uno de los más afectados, dada la paralización de las clases presenciales desde el nivel pre-básico hasta el superior y, a su vez, la suspensión o modificación de toda práctica profesional presencial en las diferentes instituciones educativas. A pesar del golpe radical en el proceso de enseñanza-aprendizaje, se han tomado medidas rápidas para dar continuidad a estos, siendo las Tecnologías de Información y la Comunicación (TIC) las herramientas más utilizadas.

El propósito de esta investigación nace ante la incertidumbre frente a la transformación metodológica provocada por la nueva modalidad virtual que se implementó en las PSP, cambiando el ritmo de trabajo de los estudiantes que forman parte de este PF. Por ello, se indaga sobre los efectos que esta modificación ha tenido en el PF de cada uno de los estudiantes que lo han vivenciado, permitiendo conocer el impacto real que ha tenido y de qué manera ha afectado el proceso como tal. Como objetivo del trabajo se estableció conocer la percepción de los estudiantes de EF de una universidad chilena frente a la práctica virtual de síntesis profesional.

\section{Método}

Se utilizó un enfoque cuantitativo no experimental transversal con alcance descriptivo, con el que se logró la recolección de datos para obtener resultados relacionados con las variables indagadas. Se logró la participa- ción de 47 estudiantes (profesores en formación) con una edad promedio de $24 \pm 1,9$ años, a quienes se les aplicó un cuestionario con 38 ítems. Las variables utilizadas fueron operacionalizadas en cinco dimensiones, a saber, datos sociodemográficos, PSP Virtual, PF, herramientas tecnológicas utilizadas durante la modalidad virtual y la valoración de la PSP en modalidad virtual. Las técnicas y los instrumentos utilizados coinciden con el enfoque dado, se utilizó la encuesta como técnica y como instrumento, con preguntas cerradas que responden a criterios de ponderación usando la escala de Likert, que va desde $1=$ totalmente en desacuerdo hasta $5=$ totalmente de acuerdo. Para la recolección de datos, se consultó a los sujetos quienes de manera voluntaria accedieron a emitir sus opiniones frente a su Prácticum virtual en Educación Física, expresando sus experiencias relacionadas con el manejo de la virtualidad al impartir sus clases de PSP y su proceso formativo. La encuesta se aplicó a estudiantes de Pedagogía en Educación Física de la Institución de Educación Superior (IES) estudiada vía correo electrónico durante los meses de agosto a septiembre del 2020. En el correo se incluyó una breve descripción de la investigación con el objetivo y un enlace utilizando la herramienta Formulario de Google. En relación con el consentimiento informado, también fue online donde los sujetos debían leer y finalmente aceptar para contestar la encuesta, esto último siguiendo los criterios éticos expuesto por el tratado de Helsinki. Para el análisis de los datos se utilizó el programa estadístico IBM SPSS 18.0 para Windows. Se trabajó con estadística descriptiva de frecuencias y porcentajes.

\section{Resultados y discusión}

Del total de sujetos participantes en este estudio, un $76,6 \%$ corresponde al género masculino mientras que un $23,4 \%$ pertenece al género femenino, entre los cuales el promedio de edad fue de 23 años, por lo que la mayoría de ellos pertenece al grupo etario de adultojoven. Respecto a las dependencias administrativas de los establecimientos educacionales donde efectuaron su PSP, un 53, 2\% de los sujetos indicó que pertenecía a una institución Municipal (escuela pública), teniendo locación en la zona urbana con un $76,6 \%$ y, a su vez, un $59.5 \%$ de ellos presentaba un índice de vulnerabilidad escolar mayor al $50 \%$, el cual es considerado como aquel que se refiere a la fragilidad de un estudiante en los distintos ámbitos sociales, corriendo posibles riesgos frente a una situación de crisis sin capacidad de poder 
afrontarla (Infante et al., 2013).

Relacionado directamente con el proceso de PSP como tal, entendido como una instancia privilegiada de socialización profesional donde el estudiante ratifica los rasgos caracterizadores del rol profesional que desempeñará en su futuro laboral (Silva et al., 2018; Anversa et al., 2020; Almonacid et al., 2021b), un 87,2\% de los estudiantes encuestados que realizaron la PSP estuvieron totalmente de acuerdo con que el proceso se llevara a cabo en una modalidad virtual.

Respecto al apoyo que recibieron por parte de aquellos que acompañan el proceso (docente guía del establecimiento educacional y el tutor de la IES), el 78,7 \% de los sujetos coincidieron en que hubo bastante apoyo por parte del profesor guía (figura 1), mas, un 21.3\% dice lo contrario. A su vez, un $91.5 \%$ mencionó un gran apoyo de parte del tutor, siendo un $8,5 \%$ quienes indican lo contrario (figura 2). Estos tienen una gran importancia tanto en el PF como en la PSP, ya que son quienes ponen a disposición sus conocimientos y experiencias, acompañando al futuro docente en su viaje formativo, brindando apoyo, retroalimentación y vinculando a estos a su futuro actuar en los establecimientos y/o centros y, a su vez, crear instancias de reflexión y autocrítica respecto a su propio desarrollo (Baeza, 2010; Hirmas, 2014; Souza de Carvalho et al., 2020). Es por esta razón que su ayuda y apoyo es fundamental dentro de procesos de esta índole y, por si fuera poco, en los nuevos escenarios para la formación virtual y/o semipresencial va a ser sustancialmente diferente al que venía ejerciendo en la enseñanza tradicional, puesto que ahora deberá desarrollar nuevas competencias (Ferrada et al., 2021).
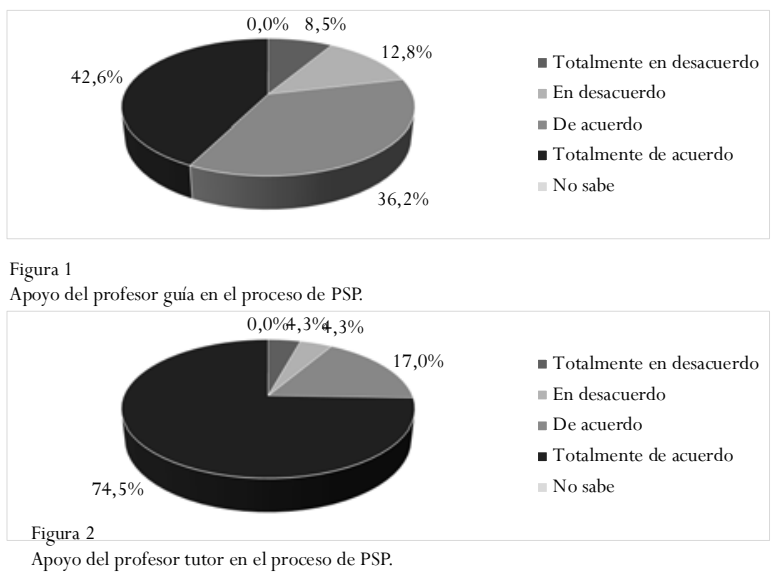

A pesar de las condiciones en las que se desarrolló la PSP en contexto de pandemia, un 93.6\% de los profesores en formación encuestados dicen sentirse preparados para enfrentar la vida laboral. No obstante, un $51 \%$ dice haber tenido mayores dificultades al efectuar el proceso de PSP en modalidad virtual (figura 3) en comparación con sus experiencias previas en aquellas prácticas tempranas que fueron realizadas de manera presencial. El cambio drástico de la presencialidad a la virtualidad en el ámbito laboral docente genera mayores dificultades (Area et al., 2018; De Vincenzi, 2020), pudiendo afectar en la satisfacción y bienestar misma del trabajador y en su misma productividad (Laca et al., 2006).

Las directrices acerca de la virtualidad suponen un reto para el quehacer docente, así como un desafío para las instituciones, más aún cuando el docente y sus estudiantes se encuentran familiarizados con un solo modelo educativo, el modelo tradicional de enseñanza-aprendizaje a través de clases magistrales, pues migrar desde este punto al modelo virtual genera sentimientos de angustia, desconfianza e incertidumbre para ambas partes (Moreno, 2020, p.17).

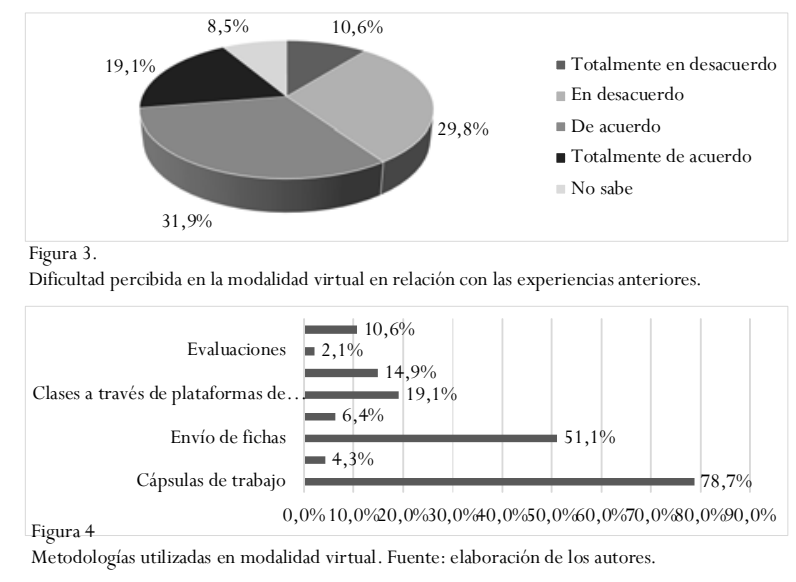

Las plataformas digitales utilizadas durante la educación virtual tienen como función facilitar al cuerpo docente la creación, administración, gestión y distribución de cursos a través de la red de Internet (Sánchez, 2009; Souza et al., 2017), «los entornos de docencia virtuales deben incorporar materiales didácticos caracterizados por presentar el conocimiento mediante lenguajes y formatos variados como son los textos, las imágenes, los audiovisuales, las animaciones» (Cabero et al., 2018, p.181). Con respecto a las metodologías virtuales utilizadas por el universo de profesores (figura 4), se pudo apreciar que las más utilizadas en esta modalidad virtual fueron las cápsulas de trabajo $(78,7 \%)$ y el envío de fichas $(51,1 \%)$.

En relación con las interacciones con los estudiantes durante el proceso de PSP en modalidad virtual, un $53,2 \%$ de ellos menciona que no tuvo interacción alguna con los estudiantes, donde solo un $29.8 \%$ del total tuvo un acercamiento por lo menos una vez a la semana y un $78.7 \%$ no tuvo atención a estudiantes fuera del horario de clases. Esto, de hecho, es sumamente pre- 
ocupante ya que estas deben ser las que permiten la comunicación para conocer los avances del proceso de aprendizaje de cada uno de los estudiantes y ejercer así su rol como mediador y facilitador del mismo (Echeverría, 2014; Araúz, 2020). Ciertamente, los entornos de aprendizaje virtuales deben ser escenarios de comunicación e interacción social permanente (Revuelta \& Pérez, 2009; Suárez \& Gros, 2013; Souza et al., 2017), mas, se ha encontrado que la tecnología digital dentro de la enseñanza ha tenido poco impacto en el aprendizaje, principalmente porque no se les otorga un sentido pedagógico a estos recursos informáticos (Cobo, 2016; Ruiz, 2020). Coincidiendo con esto, un 38,3\% de los encuestados cree que la cantidad de intercomunicaciones entre docente/estudiante no fue suficiente para la adquisición de aprendizajes significativos y un 48.9\% no tiene certeza si esto se cumplió o no.

Sin embargo, como se aprecia en la figura 5, un 40.5\% de ellos menciona que los estudiantes participaban siempre y/o regularmente de las clases planificadas, de los cuales un 80.8\% afirma que estas fueron desarrolladas de acuerdo con lo que previamente desglosaron en sus planificaciones.

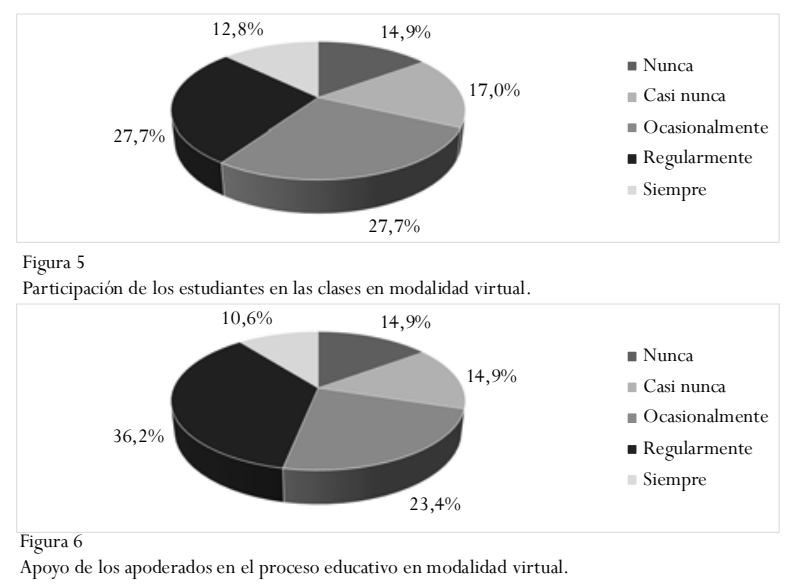

En relación con la participación de los apoderados y/o padres de los estudiantes de la comunidad educativa dentro del proceso de educación virtual (figura 6), un $87.2 \%$ de los encuestados menciona que su colaboración a este proceso fue de medio a bajo, donde un 53.2\% afirma que su colaboración tampoco fue la mejor para facilitar la PSP en modalidad virtual. Dato relevante, ya que el proceso educativo es una tarea compartida entre los docentes y los padres y/o apoderados, teniendo la responsabilidad conjunta de hacer partícipes a los estudiantes y facilitarles lo necesario para que adquieran los aprendizajes y se desenvuelvan en todo ámbito (Cabrera, 2009). El Instituto Nacional para la Evaluación de la Educación (INEE, 2003), menciona que para una educación de calidad es indispensable la interacción efectiva entre los padres de familia, los docentes y todos los sectores de la sociedad (Valdés et al., 2009).

En cuanto al PF, es aquel mediante el cual una persona o grupo de personas configura una perspectiva diferente de los contenidos, procedimientos y actitudes que ya conocían o habían adquirido previamente, esta perspectiva les permite tomar decisiones fundamentadas en todo aquello que conocen o han podido elaborar. Decimos entonces que estas personas han llevado a cabo un proceso de aprendizaje (Moreno, 2002), de ahí la importancia de establecer los principales aspectos del PF que llevaron a cabo los ya profesores. De acuerdo con los resultados (figura 7), se puede establecer que el 70,2\% presenta una positividad y considera que la IES durante sus años de formación, e independiente de la duración de esta, logró transferir a los estudiantes las competencias y herramientas necesarias para desenvolverse en todos los ambientes posibles, específicamente en la nueva modalidad online en donde estos han desarrollado su PSP, mientras que un $27.6 \%$ no se está de acuerdo con esta afirmación.

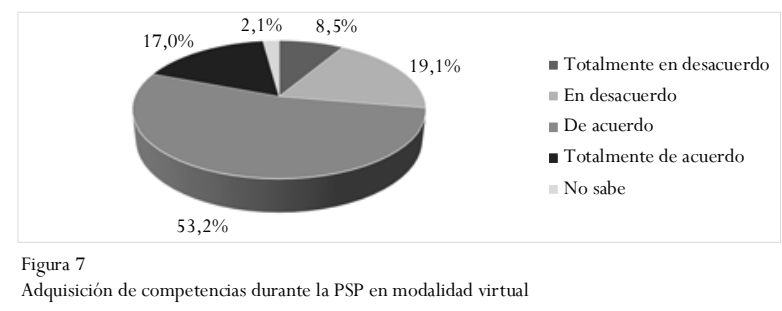

Debido a la situación sanitaria actual, el Ministerio de Educación de Chile (MINEDUC), a través de la Unidad de Currículum y Evaluación, presentó al Consejo Nacional de Educación una propuesta cuyo fin es priorizar aquellos objetivos y aprendizajes considerados imprescindibles de continuar dentro del PF de los niños, niñas y jóvenes del país durante la emergencia y la dificultad de retomar las clases presenciales (MINEDUC, 2020). De acuerdo a lo mencionado, un $95.7 \%$ de los encuestados considera que, durante la formación profesional, lograron adquirir las competencias imprescindibles para trabajar de acuerdo con la Priorización Curricular. A su vez, un 80.8\% de los encuestados considera que durante el transcurso de su formación profesional lograron adquirir las competencias necesarias para implementar diversas estrategias para el proceso de enseñanza-aprendizaje, independiente de la modalidad de las clases, vale decir, presencial o virtual.

Respecto a la relación con las herramientas tecnológicas, podemos observar que un 70,2\% de los encuestados tienen conocimientos sobre las TIC utilizadas por la IES estudiada y, dentro de este porcentaje, el 
47,2\% cree que IES se encuentra preparada para enfrentar un futuro suceso virtual, sin embargo, un 36,1\% no considera que se encuentre apta para abordar un proceso similar, porcentaje no menor (figura 8).

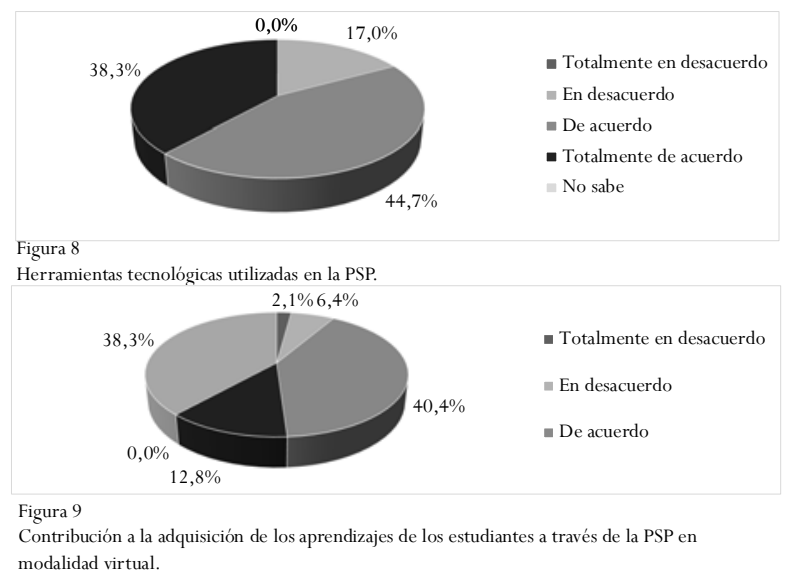

Varias universidades ya contaban con procesos para realizar clases online, pero hay otras que esto lo veían muy lejano. Debido a la actual pandemia, se tuvo que reorganizar todo el año educativo y, ante esto, la mayoría de los estudiantes chilenos se vieron afectados producto del cierre de los establecimientos educacionales a los cuales asistían, teniendo que adaptarse a un nuevo contexto educativo (Vásquez et al., 2020). Para esto, el desarrollo de habilidades digitales es sumamente necesario al igual que contar con las herramientas para ello, en Chile esto es insuficiente y no se articula con los procesos vitales de los habitantes del país (Valdivia et al., 2019).

Respecto a la valoración del profesorado en relación a la PSP de manera virtual, se trata de un proceso que debe orientarse fundamentalmente hacia la estimación de la calidad de la enseñanza, con el fin de contribuir de manera progresiva a su mejora. Este no debe verse como una estrategia de vigilancia controladora, sino como una forma de fomentar y favorecer el perfeccionamiento de los docentes, identificando las cualidades que conforman a un buen profesor (Tejedor, 2012; Cabero, et al., 2018). De acuerdo con la satisfacción de los encuestados en función de la PSP en modalidad virtual, un 31,9\% presenta un nivel de complacencia aceptable. Por otro lado, un 63.8\% considera que este proceso no tuvo mayores complicaciones, por lo que podemos aseverar que la mayoría realizó su proceso de PSP en condiciones favorables para un buen desarrollo, pudiendo así transmitir cada uno de los aprendizajes esperados.

Para un docente es fundamental contribuir en los aprendizajes de sus estudiantes y darle las herramientas necesarias para que se desenvuelvan de una manera in- tegral. La calidad de educación virtual es la adquisición de una serie de competencias, habilidades, conocimientos y destrezas por parte de los estudiantes mediante el desarrollo de contenidos de aprendizaje adecuados, impartidos a través de herramientas web eficientes (Seoane et al., 2006). Al evaluar el ámbito de la adquisición de los diferentes aprendizajes en sus estudiantes, un 53,2\% de los encuestados está de acuerdo con que tuvieron una influencia considerable en el proceso, transmitiendo los distintos conocimientos y la adquisición de los aprendizajes esperados (figura 9).

De acuerdo con la percepción de los encuestados en cuanto a la eficacia de la transmisión de los aprendizajes significativos para los estudiantes en relación con la modalidad por la cual realizaron la PSP, el 85,1\% cree que la presencialidad es fundamental para los estudiantes y la transmisión de conocimientos, frente a un 14,9\% que expresa que en la combinación de ambas modalidades se traspasan de manera efectiva, generando un aprendizaje significativo. Ciertamente, un ambiente en el que abunde comunicación, interacciones, materiales pedagógicos y una mayor expresión, hace que el aprendizaje tenga más influencia (Prieto, 2004; Flores, 2019). Según Del Hierro et al. (2014), los profesores de modalidad virtual deben poseer características fundamentales como el dominio de habilidades tecnológicas, predominando la utilización de la plataforma tecnológica y la publicación de materiales de utilidad para la asignatura en la misma plataforma, asimismo, señalan como necesario que el docente promueva la comunicación en el curso y sea un agente motivador para promover actividades que apoyan la autorregulación del aprendizaje por el propio estudiante, haciendo más fácil las clases presenciales para los procesos de enseñanza-aprendizaje.

\section{Conclusión}

La formación inicial del profesorado ha ido ascendiendo en términos de importancia, brindando así una mayor relevancia dentro del proceso de aprendizaje de los mismos, buscando que los y las docentes sean capaces de innovar dentro de las metodologías de enseñanza a través de la incorporación de una serie de herramientas digitales, las cuales contribuyen a generar un ambiente de aprendizaje renovado y contextualizado a la nueva realidad que están viviendo los niños y jóvenes pertenecientes a la educación formal. Bajo esa tesitura, se dejó en evidencia que la mayoría de los egresados sintieron que el proceso virtual llevado a cabo durante su último semestre fue un poco más difícil de lo que 
habían desarrollado en anteriores experiencias en una modalidad presencial. Pese a todo, sienten que se encuentran preparados para enfrentar la vida laboral, ya que en este contexto adquirieron nuevas competencias relacionadas al manejo de las TIC. A su vez, consideran que la IES cuenta con las herramientas necesarias para el desarrollo de las clases virtuales, pero también son imprescindibles las capacitaciones al profesorado y estudiantado para un correcto desarrollo del proceso enseñanza-aprendizaje virtual.

\section{Referencias}

Almonacid-Fierro, A., Vargas Vitoria, R., Mondaca Urrutia, J., \& Sepúlveda-Vallejos, S. (2021a). Prácticas profesionales en tiempos de pandemia Covid-19: Desafíos para la formación inicial en profesorado de Educación Física (Professional practices in times of Covid-19 pandemic: Challenges for Physical Education initial teaching training). Retos, 42, 162-171. https:/ /doi.org/10.47197/retos.v42i0.87353

Almonacid-Fierro, A., Souza de Carvalho, R., Castillo-Retamal, F. \& Almonacid, M. (2021b). The Practicum inTimes of Covid19: Knowledge Developed by Future Physical Education Teachers in Virtual Modality. International Journal of Learning, Teaching and Educational Research, 20(3), 68-83. https:// doi.org/10.26803/ijlter.20.3.5

Anversa, A. L., Souza,V.de F., Both, J., da Costa, L. C., Batista, P. \& Oliveira, A. (2020). Escala de avaliação da constituição da identidade profissional do bacharel em educação física (Assessment scale of the constitution of professional identity of the bachelor in physical education) (Escala de evaluación de la constitución de la identidad pr. Retos, 38(38), 196-203. https://doi.org/10.47197/retos.v38i38.74749

Araúz, I. (2020). El desempeño de la docencia y su desafío frente a la Educación Virtual en profesores de tres universidades de Chiriquí, Panamá. Espila, 3 (1), 16.-19. https:// revista.isaeuniversidad.ac.pa/index.php/EIL/article/view/ $56 / 60$

Area,M., San Nicolás, M. \& Sanabria,A. (2018).Las aulas virtuales en la docencia de una universidad presencial: la visión del alumnado. RIED, 21(2), 181. https://doi.org/10.5944/ ried.21.2.20666

Ávalos, B. (2002). Profesores para Chile, Historia de un proyecto. Ministerio de Educación. http://www.ub.edu/obipd/ PDF\%20docs/Aspectes\%20laborals / Documents/

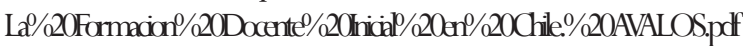

Baeza, A. (2010). Percepciones en torno al rol y las competencias del profesor guía por parte de actores educativos involucrados en la experiencia de práctica profesional docente en la región metropolitana de Santiago. OEI.

https: / / www.academia.edu / 15022129 /

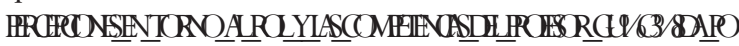

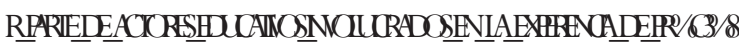

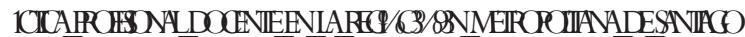

Bisconsini, C. R. \& Oliveira,A.A. B. (2018). A prática como componente curricular na formação inicial de professores de educação física. Journal of Physical Education, 24(2), 455-470. https: / / doi.org/10.22456/1982-8918.76705

Cabero, J., Llorente, M. \& Morales, J. (2018). Evaluación del desempeño docente en la formación virtual: ideas para la configuración de un modelo. Revista Iberoamericana de Educación a Distancia, 21(1), 261-279. http://dx.doi.org/10.5944/ ried.21.1.17206

Cabrera, M. (2009). La importancia de la colaboración familiaescuela en la educación. Innovación y experiencias educativas, 16, 1-9. https: / / archivos.csif.es / archivos/andalucia/ ensenanza/revistas/csicsif/revista/pdf/Numero_16/ MARIA_CABRERA_1.pdf

Castillo-Retamal, F., Almonacid-Fierro, A., Castillo-Retamal, M. \& Oliveira, A.A.B. (2020). Formación de profesores de Educación Física en Chile: una mirada histórica (Physical Education teacher training in Chile: a historical view). Retos, 38(38), 317 324. https://doi.org/10.47197/retos.v38i38.73304

Castillo-Retamal, F., Oliveira, A. A. B., Souza de Carvalho, R., Castillo-Retamal, M. \& Faúndez-Casanova, C. (2019). Competency based Physical Education teacher training: the case of a chilean university. Journal of Physical Education, 30(1), 1-10. https://doi.org/10.4025/jphyeduc.v30i1.3003

Chamorro, A., González, M. \& Gómez, A. (2008). Las prácticas pedagógicas que construyen conocimiento colectivo en el aula con estudiantes de pregrado de tres universidades de Bogotá [Tesis de Magíster, Bogotá: Universidad de La Salle]. https:// c i e n c i a . l a s a l l e. e d u . c o / c g i / viewcontent.cgi?article $=1607 \&$ context $=$ maest_docencia

Cobo, C. (2016). La Innovación Pendiente. Reflexiones (y provocaciones) sobre Educación, Tecnología y Conocimiento. Colección Fundación Ceibal. Montevideo: Debate.

https: / digital.fundacionceibal.edu.uy/jspui/bitstream/ 123456789/159/1/La_innovacion_pendiente.pdf

DeVincenzi,A. (2020). Del aula presencial al aula virtual universitaria en contexto de pandemia de COVID-19. Debate Universitario, 8(16),1-7. http://200.32.31.164:9999/ojs/ index.php/debate-universitario/article/view/238/242

Del Hierro, E., García, R. \& Mortis, S. (2014). Percepción de estudiantes universitarios sobre el perfil del profesor en la modalidad virtual-presencial. EDUTEC. Revista Electrónica de Tecnología Educativa, 48, 1-18. https://doi.org/10.21556/ edutec. 2014.48.114

Echeverría,A. (2014). Usos de lasTIC en la docencia universitaria: opinión del profesorado de educación especial. INIE, 14(3), 1-24. https://www.scielo.sa.cr/pdf/aie/v14n3/ a12v14n3.pdf

Ferrada-Bustamante, V., González-Oro, N., Ibarra-Caroca, M., Ried-Donaire, A., Vergara-Correa, D. \& Castillo-Retamal, F. (2021). Formación docente en TIC y su evidencia en tiempos de COVID-19. Revista Saberes Educativos, 6, 144-168. https: / /doi.irg/10.5354/2452-5014.2021.60715 
Flores, J. (2019). La relación docente-alumno como variable mediadora del aprendizaje. Revista San Gregorio, 35, 174-186. https://doi.org/10.36097/rsan.v1i35.957

Flores-Ferro, E., Maureira-Cid, F.,Hadweh-Briceño, M., GutiérrezDuarte, S., Silva-Salse, Á., Peña-Troncoso, S... Lagos-Olivos, C. (2021). Nivel de satisfacción de las clases online por parte de los estudiantes de Educación Física de Chile en tiempos de pandemia (Level of satisfaction of online classes by students of Physical Education of Chile in times of pandemic). Retos, (41), 123-130. https://doi.org/10.47197/retos.v0i41.82907

Hirmas, C. (2014).Tensiones y desafíos para pensar el cambio en la formación práctica de futuros profesores. Estudios pedagógicos, 40(1), 127-143. http://dx.doi.org/10.4067/S071807052014000200008

INEE. (2003). La calidad de la Educación Básica en México. Primer informe anual. Ciudad de México: Instituto Nacional para la Evaluación de laEducación.https: / /www.inee.edu.mx/wpcontent/uploads/2018/12/P1B202.pdf

Infante, M., Matus, C., Paulsen,A., Salazar,A. \&Vizcarra, R. (2013). Narrando la Vulnerabilidad Escolar: performatividad, espacio y territorio. Literatura y lingüística, 27, 281-308. https:// scielo.conicyt.cl/pdf/lyl/n27/art14.pdf

Laca, F., Mejía, J. \& Gondra, J. (2006). Propuesta de un modelo para evaluar el bienestar laboral como componente de la salud mental. Psicología y Salud, 16 (1), 87-92. https:// psicologiaysalud.uv.mx/index.php/psicysalud/article/ view/796/1444

Martínez, E. \& González, M. (2009). ¿La creatividad como competencia universitaria? La visión de los docentes. Revista de Formación e Innovación Educativa Universitaria, 2(2), 235-248. Recuperado de https://dialnet.unirioja.es/servlet/ articulo?codigo $=3110884$

MINEDUC. (2008). Marco para la buena enseñanza (7 ed.). Maval. https://www.docentemas.cl/docs/MBE2008.pdf

MINEDUC. (2020). Fundamentos Priorización Curricular Covid-19. Unidad de Currículum Evaluación. https:// www.curriculumnacional. cl / 614 / articles 179650_recurso_pdf.pdf

Moreno, P. (2002). Diseño y planificación del aprendizaje. Biblioteca Daniel Cossio Villegas. http://biblio.colmex.mx/ curso_formacion_formadores/NdC\%20de\%20Fdf.pdf

Moreno, S. (2020). La innovación educativa en los tiempos del Coronavirus. Salutem Scientia Spiritus, 6(1), 14-26. https:// pesquisa.bvsalud.org/portal/resource/pt/biblio-1087909

Prieto, D. (2004). La comunicación en la Educación. Ediciones la Crujía. https://proyectos-escolares8.webnode.es/_files/ 200000072-2aba12 cac2 / 140953778 -LIBRO-LaComunicacion-en-la-Educacion-PRIETO-CASTILLOdoc.pdf

Revuelta, F. \& Pérez, L. (2009). Interactividad en los entornos de formación on-line. UOC.

Ruiz, G. (2020). Marcas de la Pandemia: El Derecho a la Educación. Revista Internacional de Educación para la Justicia Social, 9(3), 45-59. https://doi.org/10.15366/riejs2020.9.3.003
Sánchez, J. (2009). Plataformas de EnseñanzaVirtual para Entornos Educativos. Pixel-Bit. Revista de Medios y Educación, 34, 217233. http://biblioteca.udgvirtual.udg.mx:8080/jspui/ bitstream/123456789/2672/1/ Plataformas\%20de\%20ense \%c 3\%b 1 anza\% 2 0virtual\%20para\%20entornos\%20educativos.pdf

Seoane Pardo, A. M., García Peñalvo, F. J., Bosom Nieto, Á., Fernández Recio, E. \& HernándezTovar, M. J. (2006).Tutoring on-line as quality guarantee on elearning-based lifelong learning. Definition, modalities, methodology, competences and skills. Virtual Campus 2006 Post-proceedings. Selected and Extended Papers, 186, 41-55. http://sunsite.informatik.rwth-aachen.de/ Publications/CEUR-WS/Vol-186/05.pdf

Silva Júnior, A. P., Both, J. \& Oliveira, A. A. B. de. (2018). Configurações e relações estabelecidas no estágio curricular supervisionado de Educação Física. Journal of Physical Education, 29(1), e-2937. https://doi.org/10.4025/ jphyseduc.v29i1.2937

Souza de Carvalho, R., Castillo-Retamal, M., Castillo-Retamal, F., Faúndez-Casanova, C., Oliveira, A. A. B. \& Souza, V. de F. M. (2020). Percepciones de profesores en formación sobre las prácticas pedagógicas supervisadas de educación física. Sportis, 6(3), 426-446. https://doi.org/10.17979/ sportis.2020.6.3.6643

Souza,V.deF.M.,Anversa,A. L. B., Bisconsini, C. R., Moreira,E.C. \& Oliveira,A. A. B. (2017). A utilização das TICS como meio facilitador no processo de formação continuada em um programa social esportivo. Journal of Physical Education, 28(1), 113. http://periodicos.uem.br/ojs/index.php/ RevEducFis/article/view/30087

Suárez, C. \& Gros, B. (2013). Aprender en red. De la interacción a la colaboración. UOC. https://www.researchgate.net/ $\begin{array}{llllllllllll}\mathrm{p} & \mathrm{u} & \mathrm{b} & \mathrm{l} & \mathrm{i} & \mathrm{c} & \mathrm{a} & \mathrm{t} & \mathrm{i} & \mathrm{o} & \mathrm{n} & /\end{array}$ 266576849_Suarez_Guerrero_C_Gros_Begona_201 3_Aprender_en red_de_ b_interacion_ a _ b_colbboracion_Barcelona_UOC Tejedor, F. (2012). Evaluación del desempeño docente. Revista Iberoamericana de Evaluación Educativa, 5(1), 319-327. https:/ /revistas.uam.es/riee/article/view/4447

Valdés, Á., Martín, M. \& Sánchez, P. (2009). Participación de los padres de alumnos de educación primaria en las actividades académicas de sus hijos. REDIE, 11(1), 1-17. http:// www.scielo.org.mx/pdf/redie/v11n1/v11n1a12.pdf

Valdivia, A., Brossi, L., Cabalin, C. \& Pinto, D. (2019). Alfabetizaciones y prácticas digitales desde agencias juveniles. Desafíos para la educación en Chile. Revista de Investigación Educacional Latinoamericana,56(2) 3-4. https://doi.org/10.7764/ PEL.56.2.2019.1

Vásquez, C., Ruz, F. \& Martínez, M. (2020). Recursos virtuales para la enseñanza de la estadística y la probabilidad: un aporte para la priorización curricular chilena frente a la pandemia de la COVID-19. Tangram, 3(2), 159-183. https://doi.org/ 10.30612/tangram.v3i2.12299 